\title{
Improvement in Network Lifetime for On-Demand Routing in Mobile Ad hoc Networks Using either On-Demand Recharging or Transmission Power Control or Both
}

\author{
Natarajan Meghanathan (Corresponding Author) \\ Department of Computer Science, Jackson State University \\ P. O. Box 18839, 1400 John R. Lynch Street, Jackson, MS 39217, USA \\ Tel: 01-601-979-3661 E-mail: natarajan.meghanathan@jsums.edu \\ Levon Paul Judon, Jr. \\ Department of Computer Science, Jackson State University \\ E-mail: solidp2001@yahoo.com
}

\begin{abstract}
Given a fixed energy budget for the operation of a mobile ad hoc network (MANET), on-demand recharging is the technique of charging the nodes initially with identical, but reduced energy level called the recharge quantum, and then recharging the nodes with the recharge quantum of energy whenever the energy level at a node goes below a threshold level. Transmission power control is the technique of adjusting the transmission power at a sender node depending on the distance to the receiver node. The high-level contribution of this paper is a simulation-based analysis of the network lifetime obtained for each of the following four scenarios: [a] No power control, No on-demand recharging; [b] Power control, but no on-demand recharging; [c] On-demand recharging, but no power control and [d] Both power control and on-demand recharging. Network lifetime is defined as the time of first node failure due to the exhaustion of energy level at the node and the inability to further charge the node. The on-demand routing protocols studied are: Dynamic Source Routing (DSR), Flow-Oriented Routing Protocol (FORP) and the Min-Max Battery Cost Routing (MMBCR) algorithm run on the top of DSR. We illustrate the improvement obtained in network lifetime as we transition from scenarios [a] through [d]. Simulation results illustrate that scenarios involving on-demand recharging ([c] and [d]) yield a higher network lifetime than scenarios [a] and [b]. When we operate the network with both on-demand recharging and power control, we obtain the maximum improvement in network lifetime. The percentage of the supplied energy that has been consumed in the network at the time of first node failure for each of the four scenarios and the three routing protocols is also measured to illustrate the effectiveness of on-demand recharging in maximizing the usage of the available energy budget.
\end{abstract}

Keywords: On-demand Recharging, Mobile Ad hoc Networks, Transmission Power Control, Network Lifetime, Routing Protocols

\section{Introduction}

A mobile ad hoc network (MANET) is a dynamic distributed system of wireless nodes where in the nodes move independent of each other. MANETs have several operating constraints such as: limited battery charge per node, limited transmission range per node and limited bandwidth. Routes in MANETs are often multi-hop in nature. Packet transmission or reception consumes the battery charge at a node. Nodes forward packets for their peers in addition to their own. In other words, nodes are forced to expend their battery charge for receiving and transmitting packets that are not intended for them. Given the limited energy budget for MANETs, inadvertent over usage of the energy resources of a small set of nodes at the cost of others can have an adverse impact on the node lifetime.

There exist two classes of MANET routing protocols: proactive and reactive. The reactive (also called on-demand) routing protocols tend to be more economical in terms of energy and bandwidth usage (Broch, Maltz, Johnson, Hu \& Jetcheva, 1998; Johansson, Larsson, Hedman, Mielczarek \& Degermark, 1999) in dynamically changing scenarios, characteristic of MANETs. Hence, in this paper, we restrict ourselves to the class of on-demand routing protocols. We use three on-demand routing protocols: Dynamic Source Routing (DSR - Johnson, Maltz \& Broch, 2001), Flow-Oriented Routing Protocol (FORP - Su \& Gerla, 1999) and Min-Max Battery Cost Routing (MMBCR - Toh, 
2001). DSR, FORP and MMBCR are respectively selected as representatives of the minimum-hop, stable path and power-aware routing strategies. In an earlier work (Meghanathan, 2008), it has been observed that FORP discovers the sequence of most stable (long-living) routes among the currently available MANET stable path routing protocols. DSR discovers minimum-hop routes with a lower routing overhead (Broch, Maltz, Johnson, Hu \& Jetcheva, 1998). MMBCR is a power-aware routing algorithm that attempts to equally distribute energy consumption to all the nodes in an ad hoc network and takes into consideration the available energy level at the nodes before route selection. The residual energy of a path is the minimum of the energy levels of the intermediate nodes on the path and MMBCR chooses the path with the maximum residual energy.

On-demand routing protocols discover a route between two nodes, only when required, using a Route Request-Reply cycle involving flooding of route discovery packets. An optimum route, depending on the route selection principles of the routing protocol, is discovered as a result of flooding. To reduce the route discovery overhead, on-demand routing protocols adopt a Least Overhead Routing Approach (LORA) wherein the discovered route is used as long as it exists. With LORA, all nodes are unfortunately not equally used and there is a tendency to overuse a few "centrally located" nodes (Toh, 2002). Especially, protocols that are designed to use minimum hop routes or stable long-living routes are more likely to unequally exhaust the battery charge of the nodes. Also, at low mobility, a node is highly likely to remain in a source-destination route for a sufficiently long time. In mobile ad hoc networks, the nodes (for example: laptops, personal digital assistants) typically belong to different users and hence the loss of connectivity to even one node is significant. Assuming that the failure of a node occurs only due to the node running out of its battery charge, we define network lifetime to be the time of first node failure.

Traditionally, MANET routing protocols have often been studied without recharging where in the fixed energy budget for the network is divided equally and all nodes are charged upfront with identical energy levels. Meghanathan \& Farago (2005) introduced the idea of on-demand recharging (referred here after simply as recharging) in MANETs and showed that given a fixed energy budget, supplying the energy in quanta to the nodes over time, rather than all of it initially, can yield significant improvement in network lifetime. Meghanathan \& Farago (2005) assumed the use of a fixed transmission power per hop and did not employ transmission power control. Transmission power control (Gomez \& Campbell, 2004) is a power-saving technique in which the transmission power at the sender is adjusted depending on the distance to the receiver node. If two nodes are closer to each other, then the transmission power needed to send a data packet between each other can be relatively smaller compared to the transmission power needed to send data between two nodes that are far away from each other. Transmission power control (referred here after simply as power control) is one of the commonly used energy-efficient techniques to optimize network lifetime in MANETs.

In this paper, we explore the use of both on-demand recharging and transmission power control to maximize network lifetime for on-demand MANET routing protocols. We conduct extensive simulations of DSR, FORP and MMBCR under each of the following four scenarios: [a] No power control, No recharging; [b] Power control, but no recharging; [c] Recharging, but no power control and [d] Both power control and recharging. Our objective is to study the improvement in network lifetime brought about by scenarios [b], [c] and [d] over scenario [a] and with respect to each other. We observe scenario [c] (recharging only) can often yield a network lifetime relatively larger than that has been obtained with scenario [b] (power control only). We also observe that operating the network with both recharging and power control leads to a significantly larger network lifetime compared to the other three scenarios.

The rest of the paper is organized as follows: Section 2 briefly reviews the idea of on-demand recharging for ad hoc networks. Section 3 reviews the DSR, FORP and MMBCR routing protocols. Section 4 explains the simulation environment. Section 5 illustrates the simulation results obtained for each of the four scenarios [a] through [d] and interprets them. Section 6 presents the conclusions.

\section{On-Demand Recharging}

Given a fixed energy budget for the operation of a MANET, the basic idea of on-demand recharging is to charge the nodes initially with identical, but reduced energy level called the recharge quantum, and then recharge a node only when it is about to run out of energy. On-demand recharging is basically a dynamic resource allocation strategy for networks with a common fixed supply of resources whose consumption across the network is unpredictable. A real-world analogical example would be the case of an investor with a fixed amount of money adjusting his/her investments in stock market according to the changing trends in the market value of the stocks.

The amount of energy added per recharge operation is called the recharge quantum and under a fixed energy budget, it is inversely related to the total number of recharges that could be done in the network before a node fails. The smaller the recharge quantum, the larger is the network lifetime and larger is the percentage of the fixed energy budget usefully consumed within the network before the time of first node failure. In this paper, in order to determine the maximum possible improvement in network lifetime, we use a recharge quantum of 1 Joule for every recharge operation. To avoid any change in performance and unnecessary route transitions, we do not let the energy level of a node to reach zero, before adding a recharge quantum. If the energy level at a node goes below the expiration threshold energy level, the 
node is supplied a recharge quantum of energy. The expiration threshold energy level is chosen in such a way that the node is always available for packet forwarding. We use an expiration threshold level of 0.05 Joules in our simulations.

On-demand recharging need not mean just physical recharging alone (Meghanathan \& Farago, 2005). For example, it could even emulate the on-demand deployment of sensors (from an initial fixed supply) to effectively track the movement of a mobile hotspot. A single phase uniform random distribution of the sensor nodes may unnecessarily increase the density of the network and also cannot guarantee that all hotspots are effectively covered. Sensors have to be deployed in a sequence of phases based on the information gathered from the sensors already deployed. On-demand sensor deployment can extend the lifetime of the network by effectively tracking a dynamically moving hotspot with immobile sensor nodes and at the same time maintain the density of the network within a reasonable magnitude.

\section{Review of the MANET Routing Protocols}

In this section, we provide a brief overview of the minimum-hop based Dynamic Source Routing (DSR) protocol, stability-based Flow-Oriented Routing Protocol (FORP) and the power-aware Min Max Battery Cost Routing (MMBCR) algorithm. In this paper, we implemented MMBCR on the top of DSR.

\subsection{Dynamic Source Routing Protocol}

The unique feature of DSR (Johnson, Maltz \& Broch, 2001) is source routing: data packets carry information about the route from the source to the destination in the packet header. As a result, intermediate nodes do not need to store up-to-date routing information in their forwarding tables. This avoids the need for beacon control neighbor detection packets that are used in the stability-oriented routing protocols. Route discovery is by means of the broadcast query-reply cycle. A source node $s$ wishing to send a data packet to a destination $d$, broadcasts a Route-Request (RREQ) packet throughout the network. The RREQ packet reaching a node contains the list of intermediate nodes through which it has propagated from the source node. After receiving the first RREQ packet, the destination node waits for a short time period for any more RREQ packets, then chooses a path with the minimum hop count and sends a Route-Reply Packet (RREP) along the selected path. If any RREQ is received along a path whose hop count is lower than the one on which the RREP was sent, another RREP would be sent on the latest minimum hop path discovered. To minimize the route acquisition delay, DSR lets intermediate nodes to promiscuously listen to the channel, store the learnt routes (from the RREQ and data packets) in a route cache and use these cached route information to send the RREP back to the source. We do not use this feature as promiscuous listening dominates the energy consumed at each node and DSR could still effectively function without promiscuous listening and route caching. Also, in networks of high node mobility, cached routes are more likely to become stale, by the time they are used.

\subsection{Flow-Oriented Routing Protocol (FORP)}

FORP (Su \& Gerla, 1999) utilizes the mobility and location information of nodes to approximately predict the expiration time (LET) of a wireless link. The minimum of LET values of all wireless links on a path is termed as the route expiration time (RET). The route with the maximum RET value is selected. Each node is assumed to be able to predict the LET values of each of its links with neighboring nodes based on the information regarding the current position of the nodes, velocity, the direction of movement, and transmission range. FORP assumes the availability of location-update mechanisms like Global Positioning System (GPS - Hofmann-Wellenhof, Lichtenegger \& Collins, 2004) to identify the location of the nodes and also assumes that the clocks across all nodes are synchronized. Route discovery is similar to the flooding-based query-reply cycle described in Section 3.1, with the information propagated in the RREQ packet being the predicted LET of each link in a path.

RREQ packets are propagated as described before, from the source node $s$ to the destination node $d$. The information recorded in this case by a node $j$ receiving a RREQ packet from a node $i$ is the predicted lifetime of the link $i-j$. The destination $d$ will receive several RREQ packets with the predicted link lifetimes in the paths traversed being listed. The residual expiration time of a path is the minimum of the predicted lifetimes of its constituent links. The $s-d$ path that has the maximum predicted residual lifetime is then selected. If more than one path has the same maximum predicted residual lifetime, the tie is broken by selecting the shortest (minimum hop path) of such paths.

\subsection{Min-Max Battery Cost Routing (MMBCR)}

The residual battery charge of an $s-d$ path is the minimum of the battery charges of the intermediate nodes of the path. The MMBCR algorithm (Toh, 2001) chooses the $s-d$ path with the largest residual battery charge. The route selection metric recorded in an $s-d$ path is the residual battery charge (available battery charge) of each of the intermediate nodes on the $s-d$ path through which the RREQ packet got forwarded. The residual battery charge of a path is the minimum of the residual battery charge of all the constituent intermediate nodes, other than the source and destination, of the path. From all the $s-d$ paths learnt, the destination chooses the path that has the largest residual battery charge value.

\section{Simulation Environment}

The simulations of DSR, FORP and MMBCR were conducted in ns-2: version 2.28 (Fall \& Varadhan, 2001). We used 
the implementation of DSR that comes with ns-2 and implemented FORP and MMBCR in ns-2. The network dimensions are $1000 \mathrm{~m} \times 1000 \mathrm{~m}$. The transmission range of each node is $250 \mathrm{~m}$. We vary the network density by conducting simulations with 25 nodes (low density network with an average of 5 neighbors per node) and 50 nodes (high density network with an average of 10 neighbors per node). The fixed energy budget for the low-density network and high-density network is 2500 Joules and 5000 Joules respectively. If we do not conduct any recharging (scenarios [a] and [b]), each node in these networks gets upfront 100 Joules of energy. When we conduct recharging (scenarios [c] and [d]), each node gets an initial energy of 1 Joule to start with and whenever the energy level at a node goes below the expiration threshold energy level of 0.05 Joules, the node is charged with the recharge quantum of 1 Joule, taken from the available energy budget for the network. For all the four scenarios, whenever a node needs energy and the available energy budget is zero, we declare the node has failed. Note in scenarios [a] and [b], the available energy budget is zero after the nodes are charged fully upfront (i.e., without any recharging). The time of first node failure is recorded as the network lifetime.

Besides network lifetime, we also measure the percentage of energy consumed in the network at the time of first node failure. This metric is basically the ratio, expressed in percentage, of the total energy actually consumed across all the nodes in the network to the initial fixed energy budget. This metric is especially of interest in scenarios [a] and [b] where there is no recharging. As illustrated in the performance figures 2.1 through 2.8 , one can observe that for both DSR and FORP, only $35 \%-60 \%$ of the energy supplied to the nodes have been consumed at the time of first node failure and even for MMBCR, the percentage of energy consumed at the time of first node failure is mostly within $75-80 \%$. On-demand recharging actually exploits this unfairness in the routing protocols and attempts to efficiently use the fixed energy budget only at nodes that need energy.

Traffic sources are continuous bit rate (CBR). Number of source-destination ( $s$ - $d$ ) sessions used is 15 (low traffic load) and 30 (high traffic load). The starting times of the $s-d$ sessions is uniformly distributed between 1 to 20 seconds. Data packets are 512 bytes in size; the packet sending rate is 4 data packets per second. While distributing the source-destination roles for each node, we saw to it that a node does not end up as source of more than two sessions and also not as destination for more than two sessions.

While DSR does not use beacons, FORP and MMBCR require the use of beacon control messages for neighborhood awareness. In these two protocols, beacons are periodically broadcast by a node within the transmission range to let each node advertise its presence to neighbors and learn about the neighborhood. In our simulations, beacons are exchanged for every one second. In the case of FORP, each node sends information about its location and current velocity in the beacons. Each node keeps track of the previously advertised location of its neighbor nodes. This will help to determine the direction in which the neighbor node is moving. For MMBCR, each node includes in the beacon packets information about the current battery charge available at the node.

\subsection{MAC Layer Model}

The MAC layer uses the distributed co-ordination function (DCF) of the IEEE Standard 802.11 (Bianchi, 2000) for wireless LANs. For scenarios with transmission power control, the channel negotiation is dealt as follows: the sender node transmits the Request-To-Send (RTS) packet with a transmission power corresponding to the fixed maximum transmission range of $250 \mathrm{~m}$. The receiver node on receiving the RTS packet, estimates the distance to the sender based on the strength of the signal received for the RTS packet. The receiver node includes this distance information in the Clear-To-Send (CTS) packet, which is transmitted with the transmission power that is just enough to reach the sender with signal strength above the receiving signal strength threshold of $3 * 10^{-10} \mathrm{~W}$. The sender node on receiving the CTS packet uses the distance information in the CTS packet and estimates the transmission power that would be just sufficient to send the DATA packet to the receiver node. The transmission power employed is calculated using the formula (Park \& Sivakumar, 2002; Meghanathan, 2008): $1.1182+7.2 * 10^{-11}(d)^{4}, d$-distance between the transmitter and the receiver. The receiver node upon successfully receiving the DATA packet will send an ACK packet using the transmission power that was spent to send the CTS packet. The neighbors of the receiver that had earlier received the CTS packet and the neighbors of the sender that had received the RTS packet are free to start their own channel negotiations after they receive the ACK packet within a certain time period.

The neighbor nodes of the sender that received the RTS packet and not the CTS packet within a certain time are free to start having their own transmissions while the DATA packet transmission is taking place. Similarly, the neighbors of the receiver that did not receive the RTS packet are also free to start having their own transmissions while the DATA packet transmission is taking place. Thus, transmission power control also helps us to increase the usage of bandwidth and minimize the delay in packet transmissions.

\subsection{Energy Consumption Model}

The energy consumption at a node in an ad hoc network can be divided into three categories: (i) Energy utilized for transmitting a message, (ii) Energy utilized for receiving a message and (iii) Energy utilized in idle state. It has been 
shown that in the presence of overhearing, no real optimization in the energy consumption or node lifetime can be achieved (Kim, Garcia-Luna-Aceves, Obraczka, Cano \& Manzoni, 2003). That is, the energy consumption at a node would be dominated by the energy lost when the node is in idle state (also referred to as being in the promiscuous mode). Thus, in this paper, we do not consider the energy lost in the idle state and focus only on the energy consumed during the transmission and reception of messages (the DATA packets, the MAC layer RTS-CTS-ACK packets and the periodic beacons), and the energy consumed due to route discoveries. We model the energy consumed due to broadcast traffic and point-to-point traffic as linear functions of the packet transmission time, network density, transmission and reception powers per hop. A similar linear modeling for energy consumption has also been earlier used (Feeney, 2001; Meghanathan, 2006). For simulations without transmission power control, the fixed transmission power per hop is $1.4 \mathrm{~W}$. For simulations with transmission power control, the transmission power per hop is dynamically adjusted using the formula $1.1182+7.2^{*} 10^{-11 *}(d)^{4}$, which includes power required to drive the circuit $(1.1182 \mathrm{~W})$ and transmission power from the antenna computed using the two-ray ground reflection model (Fall \& Varadhan, 2001) and distance $d$ between the sender and receiver. The reception power per hop is fixed for all situations and it is $0.967 \mathrm{~W}$. The above values/ equations for transmission and reception power per hop are commonly used (Feeney, 2001; Meghanathan, 2006; Park \& Sivakumar, 2002) in simulation studies.

\subsection{Node Mobility Model}

The node mobility model used is the Random Waypoint model (Bettstetter, Hartenstein \& Perez-Costa, 2004), a widely used mobility model in MANET simulation studies. According to this model, each node starts moving from an arbitrary location to a randomly selected destination location at a speed uniformly distributed in the range $\left[v_{\min }, \ldots, v_{\max }\right]$. Once the destination is reached, the node may stop there for a certain time called the pause time and then continue to move by choosing a different target location and a different velocity. In this paper, we set $v_{\min }=0$, and each node chooses speed uniformly distributed between 0 and $v_{\max }$. The $v_{\max }$ values used are $5 \mathrm{~m} / \mathrm{s}$ (representing low node mobility condition) and $50 \mathrm{~m} / \mathrm{s}$ (representing high node mobility condition). Pause time is 0 seconds. Note that as the node velocities for each direction change are randomly picked from $\left[\begin{array}{llll}0 & \ldots & 5 \mathrm{~m} / \mathrm{s}\end{array}\right]$ and $\left[\begin{array}{llll}0 & \ldots & 50 \mathrm{~m} / \mathrm{s}\end{array}\right]$, the average velocity per node in the low node mobility and high node mobility conditions is about $2.5 \mathrm{~m} / \mathrm{s}$ and $25 \mathrm{~m} / \mathrm{s}$ respectively. These two values translate to 6 miles per hour and 55 miles per hour representing vehicular speeds in a school environment and interstate highways respectively.

\section{Simulation Results}

Each data point in Figures 1 and 2 is an average of data collected using 5 mobility trace files and 5 sets of randomly selected 15 and $30 s-d$ sessions.

\subsection{Network Lifetime}

Considering scenarios $[\mathrm{b}]$ and $[\mathrm{c}]$, wherein only one of the two energy-efficient techniques is implemented, we observe that on-demand recharging yields a significant improvement in network lifetime compared to transmission power control. On average, when we implement only on-demand recharging (scenario [c]), DSR, FORP and MMBCR show an improvement of $86 \%, 92 \%$ and $36 \%$ respectively and when we implement only power-control (scenario [b]), DSR and FORP show an improvement of only $52 \%$ and $59 \%$ respectively. The network lifetime for MMBCR when operated with power control is even slightly lower than when operated without power control (by a factor of $12 \%$ ).

When we operate the network with both on-demand recharging and transmission power control (scenario [d]), DSR, FORP and MMBCR showed an improvement of (a) about $150-200 \%, 150-300 \%$ and $50-70 \%$ respectively compared to the scenario of operating without both on-demand recharging and transmission power control, (b) about $75-85 \%$, $75-140 \%$ and $78-100 \%$ respectively compared to the scenario of operating only with transmission power control and (c) about $35-64 \%, 54-90 \%$ and $18-20 \%$ respectively compared to the scenario of operating only with on-demand recharging. So, even though the absolute network lifetime is relatively lower, FORP incurred the maximum improvement in network lifetime and this can be attributed to the supply of energy to the nodes lying on the long-living stable paths as long as the available energy budget is positive.

When we operate the network without both power control and on-demand recharging (scenario [a]), MMBCR incurs the largest network lifetime for low-density networks and DSR incurs the largest network lifetime for high-density networks. FORP performs very poorly in this scenario for both low-density and high-density networks. FORP, being a stable path routing protocol, tends to overuse nodes lying on the stable path at the cost of others. To a certain extent, DSR also overuses nodes (especially in low-density networks) lying in the center of the network compared to nodes in the periphery.

MMBCR shows the least improvement in network lifetime and DSR and FORP show the a relatively larger improvement in network lifetime as we move from scenarios of no power control and no on-demand recharging towards scenarios of power control and on-demand recharging. This can be attributed to the power-aware nature of MMBCR. The gain in network lifetime with the introduction of the energy-efficient techniques is relatively low with MMBCR. 
On the other hand, the performance of DSR and FORP with respect to network lifetime improved as we introduce scenarios involving power control and/ or on-demand recharging. For both these non power-aware routing protocols, with on-demand recharging, nodes that are being used more frequently receive more energy and nodes that are used less frequently receive less energy. DSR preferentially uses nodes that lie in the center of the network to be part of several minimum-hop routes and FORP preferentially uses nodes maintain stable links with its neighbors to be part of several stable routes. As MMBCR inherently attempts to ensure fairness of node usage at the cost of hop count and route stability, with on-demand recharging, energy is still likely to be equally distributed and used across all the nodes.

For a given level of node mobility and network density, as we increase the offered traffic load to $30 s-d$ pairs, the network lifetime is reduced by almost half of the value observed with $15 s-d$ pairs for each of the four scenarios [a] through [d]. Nevertheless, the rate of improvement in network lifetime as we transition from scenarios [a] through [d] remains the same, irrespective of the offered traffic load.

For a given level of network density and offered traffic load, in general, for scenarios [a] and [b], the routing protocols yield a relatively lower network lifetime when operated under low mobility conditions rather than the high mobility conditions. Even though, frequent route changes result in higher energy consumption, route changes are preferred to yield diversity in the routes and achieve better fairness of node usage. But, as we transition to scenario [d], the routing protocols yield a larger network lifetime under low mobility conditions rather than high mobility conditions. Under low-mobility conditions, the energy consumed due to route discoveries is relatively low and with the introduction of on-demand recharging, nodes that are being heavily used get more energy compared to nodes that are lightly used.

For a given level of node mobility and offered traffic load, under each of the scenarios [a] through [d], we observe the network lifetime with DSR as the routing protocol increases with increase in network density (from 25 nodes to 50 nodes) and the network lifetime with FORP decreases with increase in network density. With increase in network density, the number of nodes in the center of the network increases and DSR has several candidate nodes to be in the minimum hop paths. On the other hand, FORP makes use of the increase in the number of nodes to find paths with a relatively longer lifetime compared to those discovered in low-density networks. Hence, even though the FORP route lifetime increases with increase in network density, the nodes that have been used in the stable path for a longer time, will fail early. With MMBCR (as it attempts to balance the energy consumption load across the network), the network lifetime remains almost the same or is slightly high with increase in network density, for a given level of node mobility and offered traffic load.

\subsection{Percentage of Energy Consumed in the Network}

With on-demand recharging (either in the absence or in the presence of power control, representing scenarios [c] and [d] respectively), for each of the routing protocols, we observe that almost the entire fixed energy budget is completely consumed (at least $99.5 \%$ ) by the nodes in the network. In the absence of on-demand recharging (scenarios [a] and [b]), the fixed energy budget is equally divided and all the nodes are charged upfront. Due to the stochastic nature of ad hoc networks and random node movements, many nodes are lightly used and have abundant energy left at them during the time of first node failure resulting from the exhaustion of the supplied energy.

Overall, we observe that in the absence of on-demand recharging, MMBCR has the highest percentage of energy consumed in the network and this is obtained in the absence of power control (scenario [a]). Even though the use of power control (scenario [b]) leads to increase in the network lifetime for MMBCR, the percentage of energy consumed in the network at the time for first node failure is less than that obtained in the absence of power control. On the other hand, the energy consumed in the network for DSR in the presence of power control is greater than that observed in the absence of power control. The percentage of energy used in the network remains almost the same for FORP for both the scenarios of operating with and without power control.

For a given level of node mobility and network density, for each of the scenarios [a] and [b], as we increase the offered traffic load to $30 s-d$ pairs, we observe that the percentage of energy consumed in the network remains the same for both FORP and DSR as observed for $15 s-d$ pairs. The percentage of energy consumed in the network for MMBCR at higher offered traffic load of $30 s-d$ pairs is $20 \%$ more than that incurred at a lower traffic load of $15 s-d$ pairs.

For a given level of network density and offered traffic load, the percentage of energy consumed in the network for both DSR and FORP at high node mobility conditions is about $30 \%$ and $15 \%$ more than that obtained at low node mobility conditions for scenarios [a] (no power control, no on-demand recharging) and [b] (power control, no on-demand recharging) respectively. The relative decrease in the magnitude of energy consumption with scenario [b] can be attributed to the reduction in the transmission power per hop. In the case of MMBCR, the percentage of energy consumed in the network at high node mobility conditions is about $35 \%$ more than that consumed at low node mobility conditions for both scenarios [a] and [b].

For a given level of node mobility and offered traffic load, under each of the scenarios [a] and [b], as we increase the network density from 25 to 50 nodes, we notice that the percentage of energy consumed in the network for FORP at 
high network density is about $20-25 \%$ lower than that consumed at low network density. On the other hand, for both DSR and MMBCR, the percentage of energy consumed in the network at high network density is about $5 \%$ more than that consumed at low network density.

\section{Conclusions and Future Work}

The simulation results highlight the improvement in network lifetime obtained with on-demand recharging compared to transmission power control. Due to the stochastic nature of ad hoc networks and random node movements, many nodes are lightly used and have abundant energy left at them during the time of first node failure due to exhaustion of the supplied energy. On-demand recharging actually exploits this unfairness in the routing protocols and attempts to efficiently use the fixed energy budget by providing energy only at nodes that need energy. With on-demand recharging (either in the absence or in the presence of power control representing scenarios [c] and [d] respectively), for each of the routing protocols, we observe that almost the entire fixed energy budget is completely consumed (at least 99.5\%) by the nodes in the network.

MMBCR shows the least improvement in network lifetime and DSR and FORP show a relatively larger improvement in network lifetime as we move from scenarios of no power control and no on-demand recharging towards scenarios of power control and on-demand recharging. This can be attributed to the power-aware nature of MMBCR. The gain in network lifetime with the introduction of the energy-efficient techniques is relatively low in the case of MMBCR. On the other hand, the performance of DSR and FORP with respect to network lifetime improved in scenarios involving power control and/ or on-demand recharging. For both these non power-aware routing protocols, with on-demand recharging, nodes that are being used more frequently receive more energy and nodes that are used less frequently receive less energy. DSR preferentially uses nodes that lie in the center of the network to be part of several minimum-hop routes and FORP preferentially uses nodes that maintain stable links with its neighbors to be part of several stable routes. As future work, we plan to study the improvement in the network lifetime obtained using on-demand recharging for multicast routing protocols.

\section{References}

Bettstetter, C., Hartenstein, H., and Perez-Costa, X. (2004). Stochastic Properties of the Random Waypoint Mobility Model. Wireless Networks, 10, 5, 555- 567.

Bianchi, G. (2000). Performance Analysis of the IEEE 802.11 Distributed Coordination Function. IEEE Journal of Selected Areas in Communications, 18, 3, 535 - 547.

Broch, J., Maltz, D. A., Johnson, D. B., Hu, Y. C., and Jetcheva, J. (1998). A Performance Comparison of Multi-hop Wireless Ad hoc Network Routing Protocols. Paper presented at the $4^{\text {th }}$ ACM/IEEE Conference on Mobile Computing and Networking, USA.

Fall, K. and Varadhan, K. (2001). NS-2 Notes and Documentation, The VINT Project at LBL, Xerox PARC, UCB, and USC.ISI, http://www.isis.edu/nsnam/ns.

Feeney, L. M. (2001). An Energy Consumption Model for Performance Analysis of Routing Protocols for Mobile Ad hoc Networks. Journal of Mobile Networks and Applications, 3, 6, 239 - 249.

Gomez, J., and Campbell, A. T. (2004). A Case for Variable-Range Transmission Power Control in Wireless Multi-hop Networks. Paper presented at the IEEE International Conference on Communications, Hong Kong.

Hofmann-Wellenhof, B., Lichtenegger, H., and Collins, J. (2004). Global Positioning System: Theory and Practice. $\left(5^{\text {th }}\right.$ rev. ed.) Springer.

Johansson, P., Larsson, T., Hedman, N., Mielczarek, B., and Degermark, M. (1999). Scenario-based Performance Analysis of Routing Protocols for Mobile Ad hoc Networks. Paper presented at the $5^{\text {th }}$ International Conference on Mobile Computing and Networking, USA.

Johnson, D. B., Maltz, D. A., and Broch, J. (2001). Ad hoc Networking, Addison-Wesley.

Kim, D., Garcia-Luna-Aceves, J. J., Obraczka, K., Cano, J.-C., and Manzoni, P. (2003). Routing Mechanisms for Mobile Ad hoc Networks based on the Energy Drain Rate. IEEE Transactions on Mobile Computing, 2, 2, 161 - 173.

Meghanathan, N. (2008). Exploring the Stability-Energy Consumption-Delay-Network Lifetime Tradeoff of Mobile Ad hoc Network Routing Protocols. Academy Publisher Journal of Networks, 3, 2, 17 - 28.

Meghanathan, N., and Farago, A. (2005). Maximizing Network Lifetime under Fixed Energy Budget in Ad hoc Networks. Paper presented at the $6^{\text {th }}$ IEEE-Southeast Conference, USA.

Meghanathan, N. and Farago, A. (2006). Comparison of Routing Strategies for Minimizing Energy Consumption in Mobile Ad hoc Networks. Paper presented at the $4^{\text {th }}$ Asian International Mobile Computing Conference, India.

Park, S.-J., and Sivakumar, R. (2002). Load Sensitive Transmission Power Control in Wireless Ad hoc Networks. Paper 
presented at the $4^{\text {th }}$ IEEE Global Communications Conference, Taiwan.

Su, W., and Gerla, M. (1999). IPv6 Flow Handoff in Ad hoc Wireless Networks using Mobility Prediction. Paper presented at the IEEE Global Telecommunications Conference, Brazil.

Toh, C.-K. (2001). Maximum Battery Life Routing to Support Ubiquitous Mobile Computing in Wireless Ad hoc Networks. IEEE Communications Magazine, 39, 6, 138 - 147.

Toh, C.-K. (2002). Ad hoc Mobile Wireless Networks, Prentice Hall.
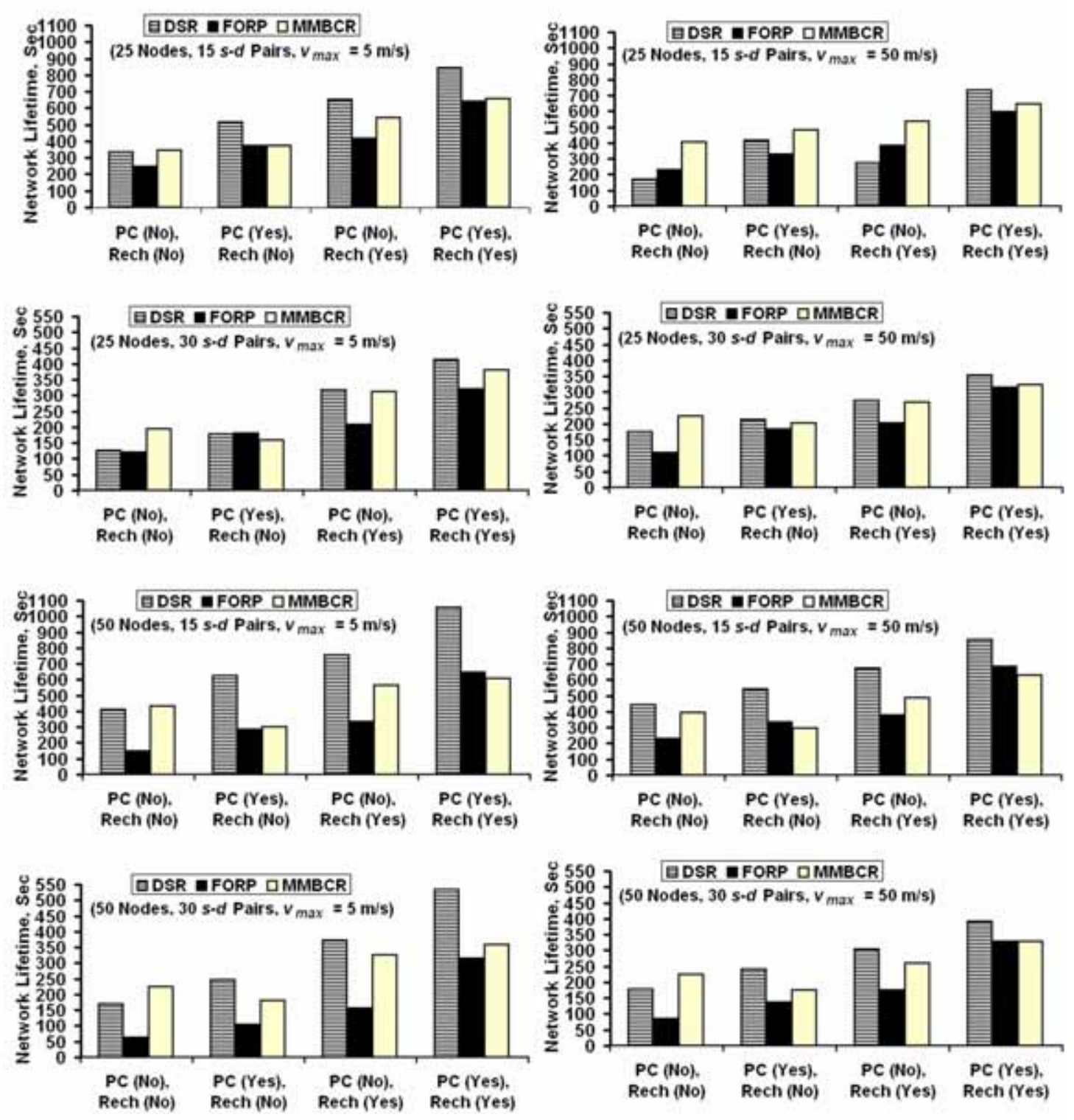

Figure 1. Network Lifetime for DSR, FORP and MMBCR 

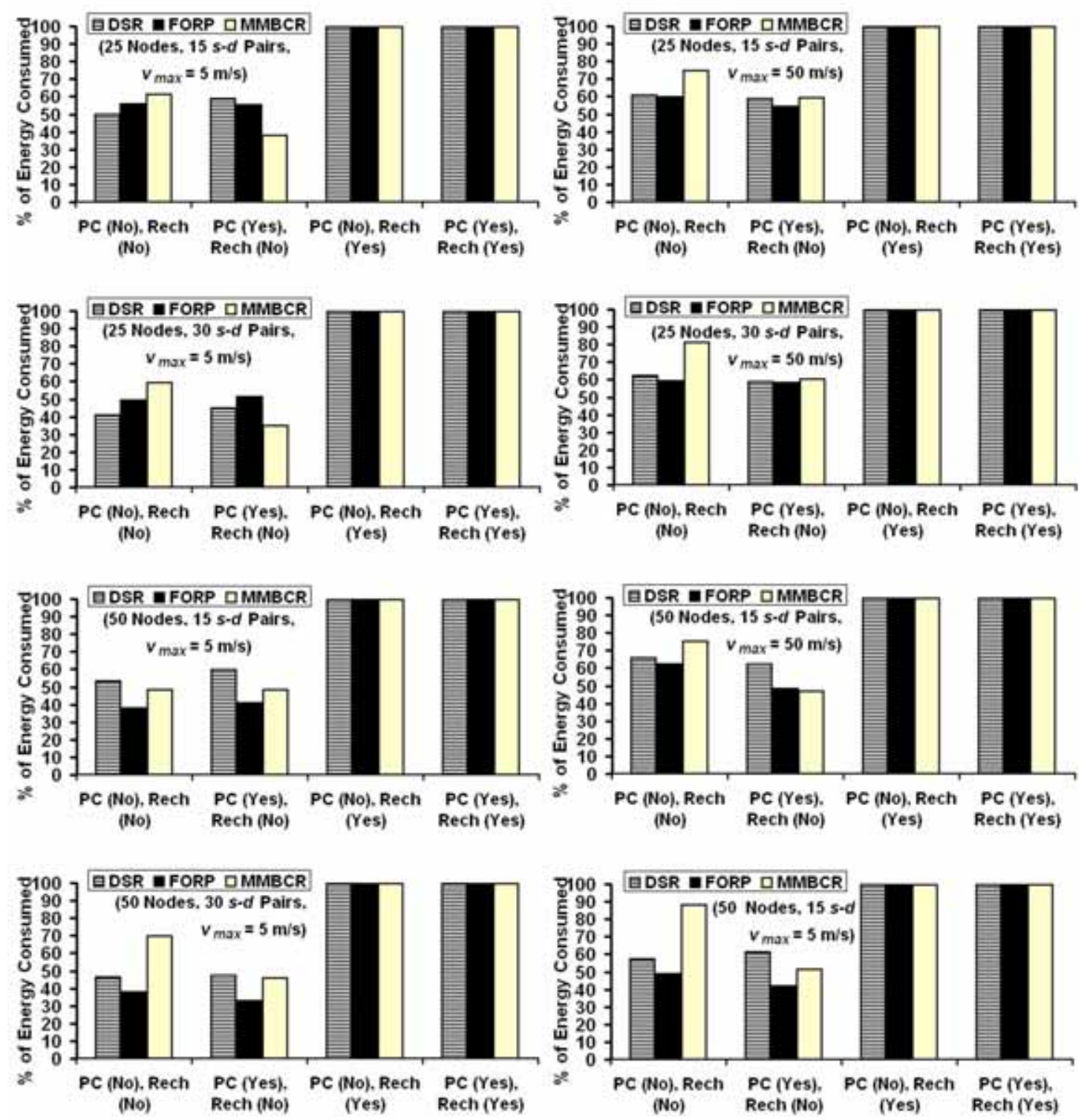

Figure 2. Percentage of the Fixed Energy Budget Actually Consumed for DSR, FORP and MMBCR 